\title{
A Review on Daphnane-Type Diterpenoids and Their Bioactive Studies
}

\author{
Yue-Xian Jin ${ }^{1,2,+}$, Lei-Ling Shi ${ }^{3,+}$, Da-Peng Zhang ${ }^{4}$, Hong-Yan Wei ${ }^{3}$, Yuan Si ${ }^{1}$, Guo-Xu Ma ${ }^{2,3, *}$ \\ and Jing Zhang ${ }^{1, *}$ \\ 1 College of Chinese Medicine Material, Jilin Agricultural University, Changchun 130118, China; \\ jyx0107@126.com (Y.-X.J.); siy2826405514@163.com (Y.S.) \\ 2 Institute of Medicinal Plant Development, Chinese Academy of Medical Sciences and PekingUnion Medical \\ College, Beijing 100193, China \\ 3 Xinjiang Institute of Chinese and Ethnic Medicine, Urumqi 830002, China; shileiling@sina.com (L.-L.S.); \\ whywlmq@sina.com (H.-Y.W.) \\ 4 College of Life Science and Technology, Xinjiang University, Urumqi 830046, China; zdp88888@yeah.net \\ * Correspondence: mgxfl8785@163.com (G.-X.M.); zhjing0701@163.com (J.Z.) \\ $\dagger$ These authors contributed equally to this work.
}

Received: 16 April 2019; Accepted: 7 May 2019; Published: 13 May 2019

check for updates

\begin{abstract}
Natural daphnane diterpenoids, mainly distributed in plants of the Thymelaeaceae and Euphorbiaceae families, usually include a 5/7/6-tricyclic ring system with poly-hydroxyl groups located at C-3, C-4, C-5, C-9, C-13, C-14, or C-20, while some special types have a characteristic orthoester motif triaxially connectedat C-9, C-13, and C-14. The daphnane-type diterpenoids can be classified into five types: 6-epoxy daphnane diterpenoids, resiniferonoids, genkwanines, 1-alkyldaphnanes and rediocides, based on the oxygen-containing functions at rings $B$ and $C$, as well as the substitution pattern of ring A. Up to now, nearly 200 daphnane-type diterpenoids have been isolated and elucidated from the Thymelaeaceae and Euphorbiaceae families. In-vitro and in-vivo experiments of these compounds have shown that they possess a wide range of biological activities, including anti-HIV, anti-cancer, anti-leukemic, neurotrophic, pesticidal and cytotoxic effects. A comprehensive account of the structural diversity is given in this review, along with the cytotoxic activities of daphnane-type diterpenoids, up to April 2019.
\end{abstract}

Keywords: daphnane; diterpenoid; cytotoxic activities

\section{Introduction}

Since the first daphnane diterpenoid characterized by a macrolactone motif was isolated from Trigonostemon reidioides [1], the daphnane diterpenoids have attracted the interest of many researchers because of their significant bioactive activities. Until now, nearly 200 natural products of daphnane-type diterpenoids have been isolated and identified, and they have shown good biological activities, including anti-HIV, anti-cancer, anti-leukemia, anti-hyperglycemic [2], neurotropic [3], insecticidal and cytotoxic [4] effects. Due to their rich pharmacological activities, especially strong anti-HIV activity and small cytotoxicity, daphnane-type diterpenoids have been employed in a range of clinical applications for a variety of clinical uses $[5,6]$. Studies have found that the natural daphnane-type diterpenoids usually embrace a 5/7/6-tricyclic ring system with poly-hydroxyl groups located at C-3, C-4, C-5, C-9, C-13, C-14, or C-20, while a special group also have a characteristic orthoester motif connected to C-9, C-13, and C-14. The daphnane-type diterpenoids can be categorized into five types (Figure 1): 6-epoxy daphnane diterpenoids, resiniferonoids, genkwanines, 1-alkyldaphnanes and rediocides, based on the substitution pattern of ring $\mathrm{A}$ and the oxygen-containing functions at rings $\mathrm{B}$ and $\mathrm{C}$. Besides, 6-epoxy 
daphnane diterpenoids usually have a C-6 $\alpha$ epoxy structure in ring B; resiniferonoids usually have an $\alpha-\beta$ unsaturated ketone structure in ring $A$; genkwanines usually have an $\alpha-\beta$ saturated ketone structure in ring $A$, but without a C-6 $\alpha$ epoxy structure in ring B; 1-alkyldaphnanes usually have a saturated ring $A$, and a large ring between the end of the orthoester alkyl chain and $C-1$ of ring $\mathrm{A}$; and rediocides usually have a 12-carbon macrolide structure between $\mathrm{C}-3$ and $\mathrm{C}-16$, and have a special C-9, C-12, and C-14 orthoester structure. The variety of daphnane-type diterpenoid structures have continued to widen with the discovery of unusual variations with the well-established skeleton. Owing to the unique skeleton and remarkable bioactive activities, daphnane-type diterpenoids have attracted many synthetic endeavors to construct a core structure. However, few papers have reported on the total synthesis of daphnane diterpenoids-isolation from natural plants is still the only source of obtaining daphnane diterpenoids. Considering the extensive interest in daphnane-type diterpenoids, we reviewed the structural and bioactive activities of daphnane-type diterpenoids, with an emphasis on the recent progress in structure identification and bioactive evaluation.

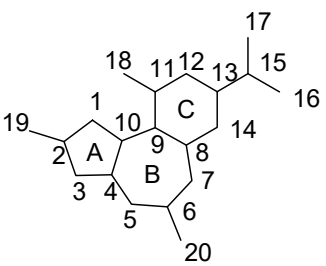

Daphnane skeleton

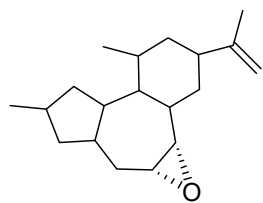
6-epoxy daphnane skeleton

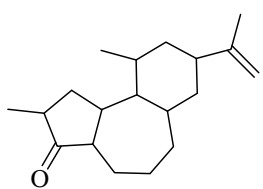

2

Resiniferonoids skeleton

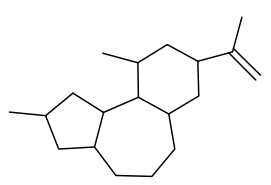

3

Genkwanines skeleton

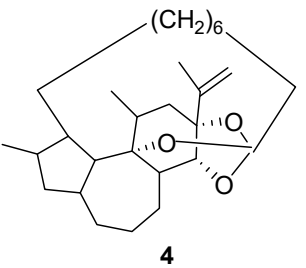

1-alkyldaphnanes skeleton

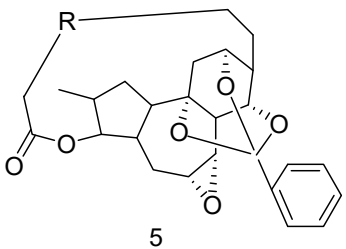

Rediocides skeleton

Figure 1. The kinds of daphnane-type diterpenoids skeleton.

\section{Occurrence}

Natural daphnane-type diterpenoids are mainly distributed in species belonging to the Thymelaeaceae or Euphorbiaceae families (Table 1). These plants grow mainly in tropical and subtropical regions of Asia [7]. Previous chemical investigations on such species have led to the isolation of a number of structurally diverse diterpenoids [8]. Various daphnane-type diterpenoids have been isolated from some parts of the following plants: The twigs and leaves of Trigonostemonthyrsoideum, the roots of Trigonostemonreidioides, the stems of Trigonostemon lii, the twigs and leaves of Trigonostemonchinensis Merr, the stem barks of Daphne giraldii, the air-dried roots of Euphorbia fischeriana, the stems of D. acutiloba, the roots of Lasiosiphonkraussianus, the flower buds of Daphne genkwa, and the roots of Maprouneaafricana Muell. Arg., Trigonostemonxyphophylloides, Wikstroemiaretusa, Trigonostemonhowii, and Stellerachamaejasme L., and so on [9]. 
Table 1. The species of daphnane-type diterpenoids.

\begin{tabular}{|c|c|c|}
\hline Types of Diterpenoids & Species & Medication Site \\
\hline $\begin{array}{l}\text { 6-epoxy daphnane } \\
\text { diterpenoids }\end{array}$ & $\begin{array}{c}\text { D. acutiloba } \\
\text { Trigonostemonthyrsoideum } \\
\text { Wikstroemiaretusa } \\
\text { Daphne genkwa } \\
\text { D. oleoidesSchreber ssp. oleoides } \\
\text { Trigonostemonxyphophylloides } \\
\text { Thymelaeahirsuta } \\
\text { Neoboutoniaglabrescens } \\
\text { S.kirkii } \\
\text { W.monticola } \\
\text { D.tangutica } \\
\text { P.elongata } \\
\text { T.xyphophylloides } \\
\text { T.thyrsoideum } \\
\text { D.vesiculosum } \\
\text { Stellerachamaejasme L. } \\
\text { Trigonostemonchinensis Merr }\end{array}$ & $\begin{array}{l}\text { Usually their effective } \\
\text { part is roots, stems, } \\
\text { twigs and leaves, } \\
\text { flower buds, fresh bark. }\end{array}$ \\
\hline Resiniferonoids & $\begin{array}{l}\text { Euphorbia fischeriana } \\
\text { Daphne genkwa } \\
\text { Euphorbia pilosa }\end{array}$ & $\begin{array}{l}\text { Generally, the roots and } \\
\text { flower budsistheir } \\
\text { effective part. }\end{array}$ \\
\hline Genkwanines & $\begin{array}{l}\text { Trigonostemonxyphophylloides } \\
\text { Trigonostemonthyrsoideum } \\
\text { Trigonostemon lii } \\
\text { Trigonostemonchinensis Merr } \\
\text { Daphne genkwa } \\
\text { Trigonostemonhowii }\end{array}$ & $\begin{array}{l}\text { Usually their effective } \\
\text { part isroots, stems, } \\
\text { twigs and leaves, } \\
\text { flower buds. }\end{array}$ \\
\hline 1-alkyldaphnanes & $\begin{array}{l}\text { Wikstroemiachamaedaphne } \\
\text { Wikstroemiaretusa } \\
\text { Stellerachamaejasme L. } \\
\text { Daphne genkwa } \\
\text { Synaptolepiskirkii } \\
\text { P.elongata }\end{array}$ & $\begin{array}{l}\text { Usually, the flower } \\
\text { buds and fresh bark is } \\
\text { their effective part. }\end{array}$ \\
\hline Rediocides & $\begin{array}{l}\text { Trigonostemonthyrsoideum } \\
\text { Trigonostemonchinensis Merr } \\
\text { Trigonostemonreidioides }\end{array}$ & $\begin{array}{l}\text { Generally, their } \\
\text { effective part is roots, } \\
\text { twigs and leaves. }\end{array}$ \\
\hline
\end{tabular}

\section{Species of Daphnane-Type Diterpenoids and Their Bioactive Activities}

\subsection{6-Epoxy DaphnaneDiterpenoids}

6-epoxy daphnane diterpenoids featurea C-6 $\alpha$ epoxy structure in ring B and, occasionally, an $\alpha-\beta$ unsaturated ketone structure in ring $A$. In most cases, there is also a $C-5 \beta$ hydroxyl group and a C-20 hydroxyl group in ring B (Figure 2, Table 2). Compounds acutilobins A-G (1-5, 65, 66), wikstroemia factor $M_{1}$ (74), genkwanineVIII (69), gniditrin (14), gnididin (15), gnidicin (13), daphnetoxin (6), yuanhuajine (50), kirkinine (24), excoecaria factor $\mathrm{O}_{1}(8)$, excoecaria toxin (7), and $14^{\prime}$-ethyltetrahydrohuratoxin(51) have been obtained from the stems of $D$. acutiloba. Acutilobins A-G have been shown to exhibit significant anti-HIV-1 activities, with $\mathrm{EC}_{50}$ below $1.5 \mu \mathrm{M}$ [10]. Trigoxyphins A (32), B (59), and trigothysoid M (63) have been isolated from the twigs and leaves of Trigonostemonthyrsoideum. These compounds have been evaluated for anti-HIV activity by an assay of the inhibition of the cytopathic effects of HIV-1 and cytotoxicity against C8166 cells. However, only trigoxyphin A expressed weak anti-HIV-1 activity [11]. Compounds huratoxin (20) and wikstroelides A-D (37-40), H-J (41-42, 56), and L-N $(43,57-58)$ have been obtained from the fresh bark of Wikstroemiaretusa. The orthoester compounds wikstroelides $\mathrm{D}$ and $\mathrm{H}$, with palmitic acid at their 20-hydroxyl site, have shown the weakest cytotoxic activity [12]. Antitumor compounds genkwanin I (64) and orthobenzoate 2 (70) have been isolated from the flower buds of Daphne genkwa. Genkwanin I has been shown to be a potent cell growth inhibitor constituent [13]. Active ingredients genkwadane 
D (9), yuanhuadine (47), yuanhuafine (45), yuanhuacine (49), yuanhuahine (44), yuanhuapine (61), genkwadaphnine (10), isoyuanhuadine (23), and genkwanine M (67) were obtained from the flower buds of Daphne genkwa. Among them, yuanhuadine, genkwadaphnine, yuanhuafine, yuanhuapine, and genkwanine $M$ have exhibited the strongest cytotoxic activities against the HT-1080 cell line $\left(\mathrm{IC}_{50}<0.1 \mu \mathrm{M}\right)$ [14]. Maprouneacin (76) has been isolated from the roots of Maprouneaafricana Muell. Arg, and has shown potent glucose-lowering properties when administered via the oral route. [15]. The compound trigonostempene C (71) has been obtained from the twigs and leaves of Trigonostemonthyrsoideum, but did not show any significant activity [16]. Compounds yuanhualine (46) and yuanhuagine (48) have been isolated from Daphne genkwa. In the analysis of signal transduction molecules, yuanhualine and yuanhuagine appear to suppress the activation of Akt, STAT3 and Src in human lung cancer cells, and also exert potent antiproliferative activity against anticancer-drug resistant cancer cells [17]. Gnidilatidin (17), gnidilatidin-20-palmitate (18), 1, 2 $\alpha$-dihydrodaphnetoxin (62), genkwadaphnin-20-palmitate (11) and gnidicin-20-palmitate (19) have successfully been obtained from the stems of D. oleoidesSchreber ssp. oleoides [18]. Trigoxyphins J and K (33-34) have been isolated from the stems of Trigonostemonxyphophylloides, and subsequently shown to be inactive against three tumor cell lines, specifically thehuman chronic myelogenous leukemia cell line (K562), the human gastric carcinoma cell line (SGC-7901), and human hepatocellular carcinoma (BEL-7402) $\left(\mathrm{IC}_{50}\right.$ value $\left.>10 \mu \mathrm{M}\right)$ [19]. Genkwanine N (68) has been obtained from the dried flower buds of Daphne genkwa, and the compound with esterification of the 20-hydroxyl has shown weak toxicity [20]. Trigonosin B (73) has beenisolated from the roots of Trigonostemonthyrsoideum [21], whilecompounds hirseins A and B (21-22) have been isolated from Thymelaeahirsuta. Hirseins A and B have shown inhibition of melanogenesis in B16 murine melanoma cells [22]. Glabrescin(12) and Montanin (26) have been obtained from Neoboutoniaglabrescens [23]. Kirkinine D (25) and synaptolepisfactor $\mathrm{K}_{7}(\mathbf{2 8})$ have been isolated from the S.kirkii [24]. Wikstrotoxin C (35) has been isolated from W.monticola. The compound 2 $\alpha$-dihydro-20-palimoyldaphnetoxin (52) has been isolated from the D.tangutica, while gnidiglaucin (16) has been obtained from P.elongata [24]. Trigoxyphin C (60) has been obtained from T.xyphophylloides, and tested against BEL-7402 cells (human hepatocellular carcinoma), where in it has been shown to be inactive ( $\mathrm{IC}_{50}$ value $>10 \mu \mathrm{M}$ was defined as inactive) [25]. Trigonosin $\mathrm{A}$ (72) has been isolated from T.thyrsoideum, and shown to exhibitin significant inhibitory activity against specific tumor cells $\left(\mathrm{IC}_{50}>10 \mu \mathrm{M}\right)$ [21]. Isovesiculosin and vesiculosin (54-55) have been isolated from D.vesiculosum [26]. Genkwanine O (75) has been obtained from D.genkwa. Compound daphnegiraldigin (53) has been isolated from the stem barks of Daphne giraldii [27]. Simplexin(27) has been obtained from Stellerachamaejasme L. [5]. Compounds trigochinins G-I (29-31) have been isolated from the twigs and leaves of Trigonostemonchinensis Merr [28].

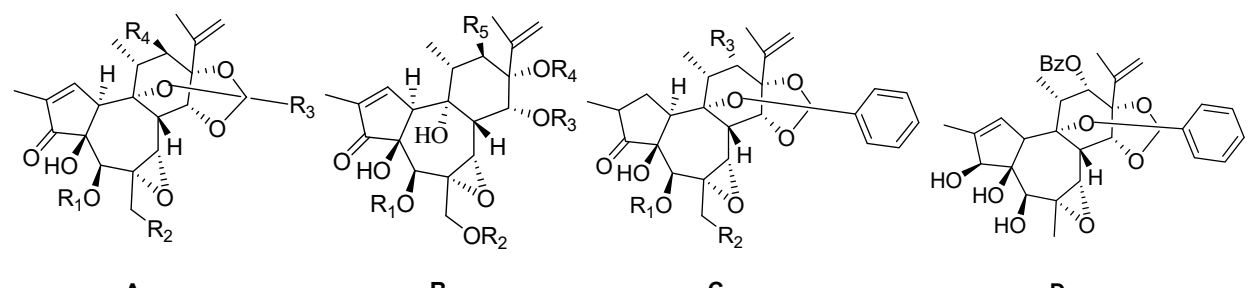

A

B

C

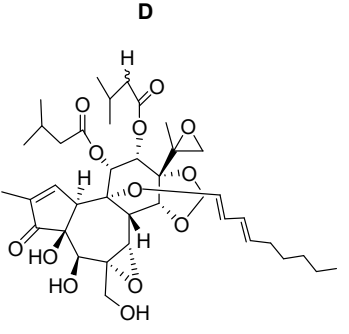

E

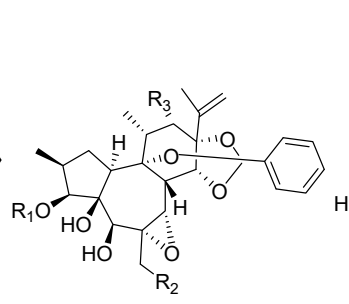

$\mathbf{F}$

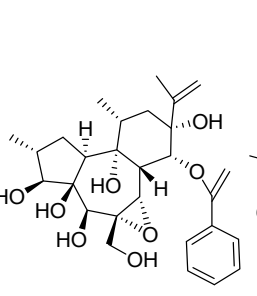

G

Figure 2. Eight types $(\mathbf{A}-\mathbf{H})$ of 6-epoxy daphnane skeletons. 
Table 2. Reported structures of 6-epoxy daphnane skeletons.

\begin{tabular}{|c|c|c|c|c|c|c|c|}
\hline No. & Name & $\mathbf{R}_{1}$ & $\mathbf{R}_{2}$ & $\mathbf{R}_{3}$ & $\mathbf{R}_{4}$ & $\mathbf{R}_{5}$ & Type \\
\hline 1 & Acutilobin A & $\mathrm{H}$ & $\mathrm{OH}$ & $\mathrm{Ph}$ & $\mathrm{OCO}(\mathrm{CH}=\mathrm{CH})_{2} \mathrm{COC}\left(\mathrm{CH}_{2}\right)_{2} \mathrm{CH}_{3}$ & - & A \\
\hline 2 & Acutilobin B & $\mathrm{H}$ & $\mathrm{OH}$ & $\mathrm{Ph}$ & $\mathrm{OCO}(\mathrm{CH}=\mathrm{CH})_{3} \mathrm{CHCH}_{2} \mathrm{CH}_{3} \mathrm{OH}$ & - & A \\
\hline 3 & Acutilobin C & $\mathrm{H}$ & $\mathrm{OH}$ & $(\mathrm{CH}=\mathrm{CH})_{3}\left(\mathrm{CH}_{2}\right)_{2} \mathrm{CH}_{3}$ & $\mathrm{OCOCH}=\mathrm{CHPhCH}_{3} \mathrm{OH}$ & - & A \\
\hline 4 & Acutilobin D & $\mathrm{H}$ & $\mathrm{OH}$ & $(\mathrm{CH}=\mathrm{CH})_{2}\left(\mathrm{CH}_{2}\right)_{4} \mathrm{CH}_{3}$ & $\mathrm{OCOCH}=\mathrm{CHPhCH} \mathrm{H}_{3} \mathrm{OH}$ & - & A \\
\hline 5 & Acutilobin E & $\mathrm{H}$ & $\mathrm{OH}$ & $\mathrm{Ph}$ & $\mathrm{OCOCH}=\mathrm{CHPhCH} \mathrm{H}_{3} \mathrm{OH}$ & - & A \\
\hline 6 & Daphnetoxin & $\mathrm{H}$ & $\mathrm{OH}$ & $\mathrm{Ph}$ & $\mathrm{H}$ & - & A \\
\hline 7 & Excoecaria toxin & $\mathrm{H}$ & $\mathrm{OH}$ & $(\mathrm{CH}=\mathrm{CH})_{2}\left(\mathrm{CH}_{2}\right)_{4} \mathrm{CH}_{3}$ & $\mathrm{H}$ & - & A \\
\hline 8 & Excoecaria factor $\mathrm{O}_{1}$ & $\mathrm{H}$ & $\mathrm{OH}$ & $(\mathrm{CH}=\mathrm{CH})_{3}\left(\mathrm{CH}_{2}\right)_{2} \mathrm{CH}_{3}$ & $\mathrm{H}$ & - & A \\
\hline 9 & Genkwadane D & $\mathrm{H}$ & $\mathrm{OH}$ & $(\mathrm{CH}=\mathrm{CH})_{2}\left(\mathrm{CH}_{2}\right)_{4} \mathrm{CH}_{3}$ & $\mathrm{OCOCH}\left(\mathrm{CH}_{3}\right)_{2}$ & - & A \\
\hline 10 & Genkwadaphnine & $\mathrm{H}$ & $\mathrm{OH}$ & $\mathrm{Ph}$ & $\mathrm{OBz}$ & - & A \\
\hline 11 & Genkwadaphnin-20-palmitate & $\mathrm{H}$ & $\mathrm{OCO}\left(\mathrm{CH}_{2}\right)_{14} \mathrm{CH}_{3}$ & $\mathrm{Ph}$ & OCOPh & - & A \\
\hline 12 & Glabrescin & $\mathrm{H}$ & $\mathrm{OCOCH}_{2}\left(\mathrm{CH}_{2}\right)_{13} \mathrm{CH}_{3}$ & $\left(\mathrm{CH}_{2}\right)_{10} \mathrm{CH}_{3}$ & $\mathrm{H}$ & - & A \\
\hline 13 & Gnidicin & $\mathrm{H}$ & $\mathrm{OH}$ & $\mathrm{Ph}$ & $\mathrm{OCOCH}=\mathrm{CHPh}$ & - & A \\
\hline 14 & Gniditrin & $\mathrm{H}$ & $\mathrm{OH}$ & $\mathrm{Ph}$ & $\mathrm{OCO}(\mathrm{CH}=\mathrm{CH})_{3}\left(\mathrm{CH}_{2}\right)_{2} \mathrm{CH}_{3}$ & - & A \\
\hline 15 & Gnididin & $\mathrm{H}$ & $\mathrm{OH}$ & $\mathrm{Ph}$ & $\mathrm{OCO}(\mathrm{CH}=\mathrm{CH})_{2}\left(\mathrm{CH}_{2}\right)_{4} \mathrm{CH}_{3}$ & - & A \\
\hline 16 & Gnidiglaucin & $\mathrm{H}$ & $\mathrm{OH}$ & $\left(\mathrm{CH}_{2}\right)_{8} \mathrm{CH}_{3}$ & OAc & - & A \\
\hline 17 & Gnidilatidin & $\mathrm{H}$ & $\mathrm{OH}$ & $(\mathrm{CH}=\mathrm{CH})_{2}\left(\mathrm{CH}_{2}\right)_{4} \mathrm{CH}_{3}$ & OCOPh & - & A \\
\hline 18 & Gnidilatidin-20-palmitate & $\mathrm{H}$ & $\mathrm{OCO}\left(\mathrm{CH}_{2}\right)_{14} \mathrm{CH}_{3}$ & $(\mathrm{CH}=\mathrm{CH})_{2}\left(\mathrm{CH}_{2}\right)_{4} \mathrm{CH}_{3}$ & OCOPh & - & A \\
\hline 19 & Gnidicin-20-palmitate & $\mathrm{H}$ & $\mathrm{OCO}\left(\mathrm{CH}_{2}\right)_{14} \mathrm{CH}_{3}$ & $\mathrm{Ph}$ & $\mathrm{OCOCH}=\mathrm{CHPh}$ & - & A \\
\hline 20 & Huratoxin & $\mathrm{H}$ & $\mathrm{OH}$ & $(\mathrm{CH}=\mathrm{CH})_{2}\left(\mathrm{CH}_{2}\right)_{8} \mathrm{CH}_{3}$ & $\mathrm{H}$ & - & A \\
\hline 21 & Hirsein A & $\mathrm{H}$ & $\mathrm{OH}$ & $\mathrm{CH}=\mathrm{CH}\left(\mathrm{CH}_{2}\right)_{4} \mathrm{CH}_{3}$ & $\mathrm{OCOCH}=\mathrm{CHPh}$ & - & A \\
\hline 22 & Hirsein B & $\mathrm{H}$ & $\mathrm{OH}$ & $\mathrm{CH}=\mathrm{CH}\left(\mathrm{CH}_{2}\right)_{4} \mathrm{CH}_{3}$ & $\mathrm{OCOCH}=\mathrm{CHPhOH}$ & - & A \\
\hline 23 & Isoyuanhuadine & $\mathrm{H}$ & $\mathrm{OH}$ & $(\mathrm{CH}=\mathrm{CH})_{2}\left(\mathrm{CH}_{2}\right)_{4} \mathrm{CH}_{3}$ & OAc & - & A \\
\hline 24 & Kirkinine & $\mathrm{H}$ & $\mathrm{OH}$ & $\mathrm{CH}=\mathrm{CH}\left(\mathrm{CH}_{2}\right)_{12} \mathrm{CH}_{3}$ & OAc & - & A \\
\hline 25 & Kirkinine D & $\mathrm{H}$ & $\mathrm{OH}$ & $(\mathrm{CH}=\mathrm{CH})_{3}\left(\mathrm{CH}_{2}\right)_{2} \mathrm{CH}_{3}$ & OAc & - & A \\
\hline 26 & Montanin & $\mathrm{H}$ & $\mathrm{OH}$ & $\left(\mathrm{CH}_{2}\right)_{10} \mathrm{CH}_{3}$ & $\mathrm{H}$ & - & A \\
\hline 27 & Simplexin & $\mathrm{H}$ & $\mathrm{OH}$ & $\left(\mathrm{CH}_{2}\right)_{8} \mathrm{CH}_{3}$ & $\mathrm{H}$ & - & A \\
\hline 28 & Synaptolepisfactor $\mathrm{K}_{7}$ & $\mathrm{H}$ & $\mathrm{OH}$ & $\mathrm{CH}=\mathrm{CH}\left(\mathrm{CH}_{2}\right)_{12} \mathrm{CH}_{3}$ & $\mathrm{H}$ & - & A \\
\hline 29 & Trigochinin G & $\mathrm{H}$ & $\mathrm{H}$ & $\mathrm{Ph}$ & $\mathrm{OCOCH}_{2} \mathrm{CH}\left(\mathrm{CH}_{3}\right)_{2}$ & - & A \\
\hline 30 & Trigochinin $\mathrm{H}$ & $\mathrm{H}$ & $\mathrm{H}$ & $\mathrm{Ph}$ & $\mathrm{OCOC}_{6} \mathrm{H}_{4}(4-\mathrm{OH})$ & - & A \\
\hline 31 & Trigochinin I & $\mathrm{H}$ & $\mathrm{H}$ & $\mathrm{Ph}$ & $\mathrm{OCOC}_{6} \mathrm{H}_{3}\left({ }_{3}-\mathrm{OMe}\right)(4-\mathrm{OH})$ & - & A \\
\hline 32 & Trigoxyphin A & $\mathrm{H}$ & $\mathrm{H}$ & $\mathrm{Ph}$ & $\mathrm{OBz}$ & - & A \\
\hline 33 & Trigoxyphin J & $\mathrm{H}$ & $\mathrm{OH}$ & $\mathrm{CH}_{3}$ & $\mathrm{OCO}\left(\mathrm{CH}_{2}\right)_{14} \mathrm{CH}_{3}$ & - & A \\
\hline 34 & Trigoxyphin $\mathrm{K}$ & $\mathrm{H}$ & $\mathrm{H}$ & $\mathrm{Ph}$ & $\mathrm{OBz}$ & - & A \\
\hline 35 & Wikstrotoxin C & & $\mathrm{OH}$ & $(\mathrm{CH}=\mathrm{CH})_{2}\left(\mathrm{CH}_{2}\right)_{4} \mathrm{CH}_{3}$ & OAc & - & A \\
\hline 36 & Wikstrotoxin D & $\mathrm{H}$ & $\mathrm{OH}$ & $\mathrm{n}-\mathrm{C}_{9} \mathrm{H}_{19}$ & $\mathrm{H}$ & - & A \\
\hline
\end{tabular}


Table 2. Cont

\begin{tabular}{|c|c|c|c|c|c|c|c|}
\hline No. & Name & $\mathbf{R}_{1}$ & $\mathbf{R}_{2}$ & $\mathbf{R}_{3}$ & $\mathbf{R}_{4}$ & $\mathbf{R}_{5}$ & Type \\
\hline 37 & Wikstroelide A & $\mathrm{H}$ & $\mathrm{OH}$ & $(\mathrm{CH}=\mathrm{CH})_{2}\left(\mathrm{CH}_{2}\right)_{8} \mathrm{CH}_{3}$ & OAc & - & A \\
\hline 38 & Wikstroelide B & $\mathrm{H}$ & $\mathrm{OH}$ & $(\mathrm{CH}=\mathrm{CH})_{2}\left(\mathrm{CH}_{2}\right)_{9} \mathrm{CH}_{3}$ & OAc & - & A \\
\hline 39 & Wikstroelide C & $\mathrm{H}$ & O-trans-5-pentadecenoic acid & $(\mathrm{CH}=\mathrm{CH})_{2}\left(\mathrm{CH}_{2}\right)_{8} \mathrm{CH}_{3}$ & OAc & - & A \\
\hline 40 & Wikstroelide D & $\mathrm{H}$ & O-palmitic acid & $(\mathrm{CH}=\mathrm{CH})_{2}\left(\mathrm{CH}_{2}\right)_{8} \mathrm{CH}_{3}$ & OAc & - & A \\
\hline 41 & Wikstroelide $\mathrm{H}$ & $\mathrm{H}$ & $\mathrm{OH}$ & $(\mathrm{CH}=\mathrm{CH})_{2}\left(\mathrm{CH}_{2}\right)_{6} \mathrm{CH}_{3}$ & OAc & - & A \\
\hline 42 & Wikstroelide I & $\mathrm{H}$ & O-palmitic acid & $(\mathrm{CH}=\mathrm{CH})_{2}\left(\mathrm{CH}_{2}\right)_{9} \mathrm{CH}_{3}$ & OAc & - & A \\
\hline 43 & Wikstroelide L & $\mathrm{H}$ & $\mathrm{OH}$ & $(\mathrm{CH}=\mathrm{CH})_{2}\left(\mathrm{CH}_{2}\right)_{8} \mathrm{CH}_{3}$ & OAc & - & A \\
\hline 44 & Yuanhuahine & $\mathrm{H}$ & $\mathrm{OH}$ & $(\mathrm{CH}=\mathrm{CH})_{2}\left(\mathrm{CH}_{2}\right)_{4} \mathrm{CH}_{3}$ & $\mathrm{OCOCH}_{2} \mathrm{CH}_{3}$ & - & A \\
\hline 45 & Yuanhuafine & $\mathrm{H}$ & $\mathrm{H}$ & $\mathrm{Ph}$ & OAc & - & A \\
\hline 46 & Yuanhualine & $\mathrm{H}$ & $\mathrm{OH}$ & $(\mathrm{CH}=\mathrm{CH})_{2}\left(\mathrm{CH}_{2}\right)_{4} \mathrm{CH}_{3}$ & $\mathrm{OCO}\left(\mathrm{CH}_{2}\right)_{2} \mathrm{CH}_{3}$ & - & A \\
\hline 47 & Yuanhuadine & $\mathrm{H}$ & $\mathrm{OH}$ & $(\mathrm{CH}=\mathrm{CH})_{2}\left(\mathrm{CH}_{2}\right)_{4} \mathrm{CH}_{3}$ & OAc & - & A \\
\hline 48 & Yuanhuagine & $\mathrm{H}$ & $\mathrm{OH}$ & $(\mathrm{CH}=\mathrm{CH})\left(\mathrm{CH}_{2}\right)_{2} \mathrm{CH}_{3}$ & $\mathrm{OCOCH}_{3}$ & - & A \\
\hline 49 & Yuanhuacine & $\mathrm{H}$ & $\mathrm{OH}$ & $(\mathrm{CH}=\mathrm{CH})_{2}\left(\mathrm{CH}_{2}\right)_{4} \mathrm{CH}_{3}$ & $\mathrm{OBz}$ & - & A \\
\hline 50 & Yuanhuajine & $\mathrm{H}$ & $\mathrm{OH}$ & $(\mathrm{CH}=\mathrm{CH})_{3}\left(\mathrm{CH}_{2}\right)_{2} \mathrm{CH}_{3}$ & $\mathrm{OBz}$ & - & A \\
\hline 51 & $14^{\prime}$-ethyltetrahydrohuratoxin & $\mathrm{H}$ & $\mathrm{OH}$ & $\left(\mathrm{CH}_{2}\right)_{14} \mathrm{CH}_{3}$ & $\mathrm{H}$ & - & A \\
\hline 52 & $2 \alpha$-dihydro-20-palimoyldaphnetoxin & $\mathrm{H}$ & $\mathrm{OH}$ & $\mathrm{CH}=\mathrm{CH}\left(\mathrm{CH}_{2}\right)_{6} \mathrm{CH}_{3}$ & OAc & - & A \\
\hline 53 & Daphnegiraldigin & $\mathrm{H}$ & $\mathrm{OH}$ & $\mathrm{COPh}$ & $\mathrm{H}$ & $\mathrm{H}$ & B \\
\hline 54 & Isovesiculosin & Ac & Ac & Ac & $\mathrm{CO}(\mathrm{CH}=\mathrm{CH})_{2}\left(\mathrm{CH}_{2}\right)_{4} \mathrm{CH}_{3}$ & $\mathrm{H}$ & B \\
\hline 55 & Vesiculosin & $\mathrm{H}$ & $\mathrm{H}$ & $\mathrm{CO}(\mathrm{CH}=\mathrm{CH})_{2}\left(\mathrm{CH}_{2}\right)_{4} \mathrm{CH}_{3}$ & $\mathrm{H}$ & $\mathrm{H}$ & B \\
\hline 56 & Wikstroelide J & $\mathrm{H}$ & $\mathrm{H}$ & $\mathrm{CO}(\mathrm{CH}=\mathrm{CH})_{2}\left(\mathrm{CH}_{2}\right)_{8} \mathrm{CH}_{3}$ & $\mathrm{H}$ & OAc & B \\
\hline 57 & Wikstroelide M & $\mathrm{H}$ & $\mathrm{H}$ & $\mathrm{CO}(\mathrm{CH}=\mathrm{CH})_{2}\left(\mathrm{CH}_{2}\right)_{8} \mathrm{CH}_{3}$ & $\mathrm{H}$ & $\mathrm{H}$ & B \\
\hline 58 & Wikstroelide N & $\mathrm{H}$ & $\mathrm{H}$ & $\mathrm{CO}(\mathrm{CH}=\mathrm{CH})_{2}\left(\mathrm{CH}_{2}\right)_{9} \mathrm{CH}_{3}$ & $\mathrm{H}$ & $\mathrm{H}$ & B \\
\hline 59 & Trigoxyphin B & $\mathrm{H}$ & $\mathrm{H}$ & $\mathrm{OBz}$ & - & - & C \\
\hline 60 & Trigoxyphin C & Ac & $\mathrm{H}$ & OBz & - & - & $\mathrm{C}$ \\
\hline 61 & Yuanhuapine & $\mathrm{H}$ & $\mathrm{OH}$ & OAc & - & - & $\mathrm{C}$ \\
\hline 62 & $1,2 \alpha$-dihydrodaphnetoxin & $\mathrm{H}$ & $\mathrm{OH}$ & $\mathrm{H}$ & - & - & $\mathrm{C}$ \\
\hline 63 & Trigothysoid M & - & - & - & - & - & $\mathrm{D}$ \\
\hline 64 & Genkwanin I & - & - & - & - & - & $\mathrm{E}$ \\
\hline 65 & Acutilobin F & $\mathrm{CO}(\mathrm{CH}=\mathrm{CH})_{3}\left(\mathrm{CH}_{2}\right)_{2} \mathrm{CH}_{3}$ & $\mathrm{OH}$ & $\mathrm{H}$ & - & - & $\mathrm{F}$ \\
\hline 66 & Acutilobin G & $\mathrm{COCH}=\mathrm{CHPh}$ & $\mathrm{OH}$ & $\mathrm{H}$ & - & - & $\mathrm{F}$ \\
\hline 67 & Genkwanine M & $\mathrm{H}$ & $\mathrm{OBz}$ & $\mathrm{H}$ & - & - & $\mathrm{F}$ \\
\hline 68 & Genkwanine N & $\mathrm{Bz}$ & $\mathrm{OH}$ & $\mathrm{H}$ & - & - & $\mathrm{F}$ \\
\hline 69 & GenkwanineVIII & $\mathrm{COPh}$ & $\mathrm{OH}$ & $\mathrm{H}$ & - & - & $\mathrm{F}$ \\
\hline 70 & Orthobenzoate 2 & $\mathrm{H}$ & $\mathrm{OH}$ & $\mathrm{H}$ & - & - & $\mathrm{F}$ \\
\hline 71 & Trigonostempene C & $\mathrm{H}$ & $\mathrm{H}$ & $\mathrm{OH}$ & - & - & $\mathrm{F}$ \\
\hline 72 & Trigonosin A & $\mathrm{H}$ & $\mathrm{H}$ & $\mathrm{OBz}$ & - & - & $\mathrm{F}$ \\
\hline 73 & Trigonosin B & $\mathrm{H}$ & $\mathrm{OH}$ & $\mathrm{OBz}$ & - & - & $\mathrm{F}$ \\
\hline 74 & Wikstroemia factor $\mathrm{M}_{1}$ & $\mathrm{CO}(\mathrm{CH}=\mathrm{CH})_{2}\left(\mathrm{CH}_{2}\right)_{4} \mathrm{CH}_{3}$ & $\mathrm{OH}$ & $\mathrm{H}$ & - & - & $\mathrm{F}$ \\
\hline 75 & Genkuanine O & - & - & - & - & - & G \\
\hline 76 & Maprouneacin & - & - & - & - & - & $\mathrm{H}$ \\
\hline
\end{tabular}




\subsection{Resiniferonoids}

Relative to 6-epoxy daphnane diterpenoids, there is no C-6 $\alpha$ epoxy structure in ring B forresiniferonoids. However, resiniferonoids do possess an $\alpha-\beta$ unsaturated ketone structure in ring A (Figure 3, Table 3). Compounds $4 \beta, 9 \alpha$, 20- trihydroxy- 13 , 15- secotiglia- 1,6- diene- 3,13- dione 20-O- $\beta$-D- [6-galloyl] glucopyranoside (86) and euphopiloside A (84) have beenisolated from the air-dried roots of Euphorbia fischeriana, and display moderate inhibitory effects against $\alpha$-glucosidase in in-vitro bioassays [29]. Yuanhuatine (78) has been isolated from the flower buds of Daphne genkwa [14]. Compounds daphneresiniferins A and B (80-81) have been obtained from the flower buds of Daphne genkwa. A study found that daphneresiniferin A was able to dependently inhibit melanin production [30]. Genkwanine L (77) has been isolated from the bud of Daphne genkwa [31]. Euphopiloside B (83), langduin A (85) and phorbol (87) have been obtained from the Euphorbia Pilosa [32], while compounds genkwadane A (79) and yuanhuaoate B (82) have been isolated from the flower buds of Daphne genkwa [14].

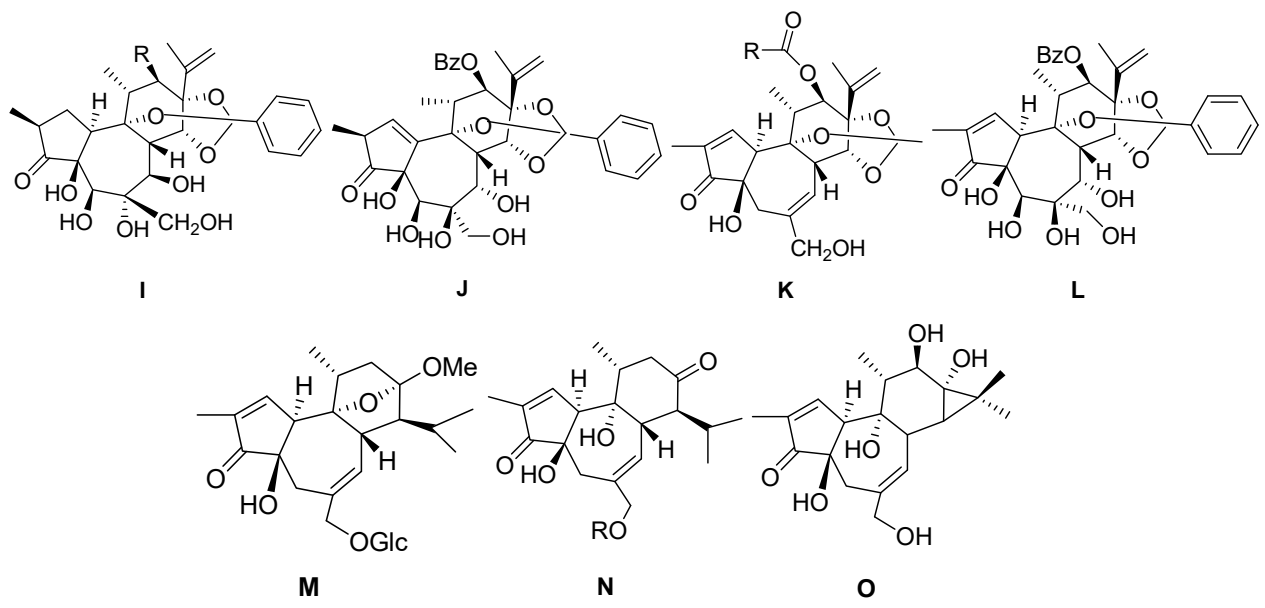

Figure 3. Seven types (I-O) of resiniferonoids skeletons.

Table 3. Reported structures of resiniferonoids skeletons.

\begin{tabular}{|c|c|c|c|}
\hline No. & Name & $\mathbf{R}$ & Type \\
\hline 77 & Genkwanine L & OAc & I \\
\hline 78 & Yuanhuatine & $\mathrm{OBz}$ & I \\
\hline 79 & Genkwadane A & - & $\mathrm{J}$ \\
\hline 80 & Daphneresiniferin A & $\mathrm{Me}$ & $\mathrm{K}$ \\
\hline 81 & Daphneresiniferin B & $\mathrm{Ph}$ & K \\
\hline 82 & Yuanhuaoate B & - & $\mathrm{L}$ \\
\hline 83 & Euphopiloside B & - & M \\
\hline 84 & Euphopiloside A & & $\mathrm{N}$ \\
\hline 85 & Langduin A & $\mathrm{H}$ & $\mathrm{N}$ \\
\hline 86 & $\begin{array}{c}4 \beta, 9 \alpha, 20 \text {-trihydroxy-13,15- } \\
\text { secotiglia-1,6-diene-3,13- } \\
\text { dione20-O- } \beta \text {-D-[6- } \\
\text { galloyl]glu-copyranoside }\end{array}$ & & $\mathrm{N}$ \\
\hline 87 & Phorbol & - & $\mathrm{O}$ \\
\hline
\end{tabular}

\subsection{Genkwanines}

Relative to 6-epoxy daphnane diterpenoids and resiniferonoids, genkwanines have an $\alpha-\beta$ saturated ketone structure in ring A, but do not possess a C-6 $\alpha$ epoxy structure in ring B (Figure 4, Table 4). Compound trigoxyphin $\mathrm{H}(\mathbf{1 0 0})$ has been isolated from the twigs of Trigonostemonxyphophylloides [33]. The active ingredients trigothysoids A-L (122-124, 96-99, 139-141, 131,128), trigochinins A-E (145-146, 130, 
147-148), andtrigonothyrins D, E (143-144) and G (121) have been obtained from the twigs and leaves of Trigonostemonthyrsoideum. These compounds have been evaluated for their anti-HIV activity usingan assay to determine their inhibition of the cytopathic effects of HIV-1 and their cytotoxicity against C8166 cells. Amongst them, trigothysoid A and L exhibited moderate anti-HIV-1 activity; andtrigothysoid C and $\mathrm{K}$ andtrigochinins $\mathrm{A}, \mathrm{B}$ and $\mathrm{D}$ expressed weak anti-HIV-1 activity [11]. Trigolins A-G (132-138) and trigonothyrin F (107) have been isolated from the stems of Trigonostemon lii. Trigolins A, G, H, and $\mathrm{K}$ have been shown to exhibit modest anti-HIV-1 activity with $\mathrm{EC}_{50}$ values of 2.04, 9.17, 11.42, and $9.051 \mu \mathrm{g} / \mathrm{mL}$, respectively [34]. Compound trigochinin F (149) has been obtained from the twigs and leaves of Trigonostemonchinensis Merr, and has shown strong inhibition of HL-60 tumor cell lines [28]. Trigonothyrins A-C (125-127) have been isolated from the stems of Trigonostemonthyrsoideum [6]. Among them, trigonothyrin $\mathrm{C}$ has shown significant activity to prevent the cytopathic effects of HIV-1 in C8166 cells, with an $\mathrm{EC}_{50}$ value of $2.19 \mu \mathrm{g} / \mathrm{mL}$ [35]. Compounds genkwanines F, I, and J $(93,113,114)$ have been isolated from the flower buds of Daphne genkwa [14]. Genkwanine H (95) has been obtained from the flower buds of Daphne genkwa, and the compound has been shown to dependently inhibit melanin production [30]. Compounds trigonostempenes A (150) and B (129) have been isolated from the twigs and leaves of Trigonostemonthyrsoideum. Studies have shown that the discovery of these NO inhibitory daphnane diterpenoids - including compound trigonostempene A-which possess $\mathrm{IC}_{50}$ values comparable topositive controls may have the potential to be developed as anti-neuroinflammatory agents for alzheimer disease (AD) and other related neurological disorders [16]. Most inhibitors of acetylcholinesterase (AchE) are alkaloids that often possess several side effects, whereas these daphnane-type diterpenoids do not belong to the class of alkaloids, and therefore they may constitute novel active AChE inhibitors with fewer side effects. It is important to search for new AChE inhibitors not belonging to this structural class [36,37]. Genkwanines A-E (88-92), G (94), I (113), and K (115) have been obtained from the bud of Daphne genkwa. Among these compounds, genkwanine $\mathrm{D}$ has been shown to exhibit strong activity to inhibit the endothelium cell HMEC at $\mathrm{IC}_{50}$ levels of 2.90-15.0 $\mu \mathrm{M}$ [31]. Compounds trigoxyphins $\mathrm{U}$ and $\mathrm{W}$ (105-116) have been isolated from the twigs of Trigonostemonxyphophylloides. Trigoxyphin W has shown modest cytotoxicity against BEL-7402, SPCA-1 and SGC-7901, with $\mathrm{IC}_{50}$ values of 5.62, 16.79 and $17.19 \mu \mathrm{M}$, respectively [33]. Trigonosins C-D $(\mathbf{1 0 6}, \mathbf{1 4 2})$ have been obtained from the roots of Trigonostemonthyrsoideum [21]. Trigoxyphin I (104) has been isolated from the Trigonostemonxyphophylloides [38]. Compounds trigohownins D and E (101-102), and trigohownins A-C (108-110) and F-I (117-120) have been obtained from the Trigonostemonhowii. Among them, trigohownins $\mathrm{A}$ and $\mathrm{D}$ have been shown to exhibit moderate cytotoxic activity against the HL-60 tumor cellline, with $\mathrm{IC}_{50}$ values of 17.0 and $9.3 \mu \mathrm{M}$, respectively [39]. Trigoxyphins D-F (111-112, 103) have been isolated from Trigonostemonxyphophylloides, with all three compounds found to be inactive against BEL-7402 cells $\left(\mathrm{IC}_{50}\right.$ value $\left.>10 \mu \mathrm{M}\right)$ [25].
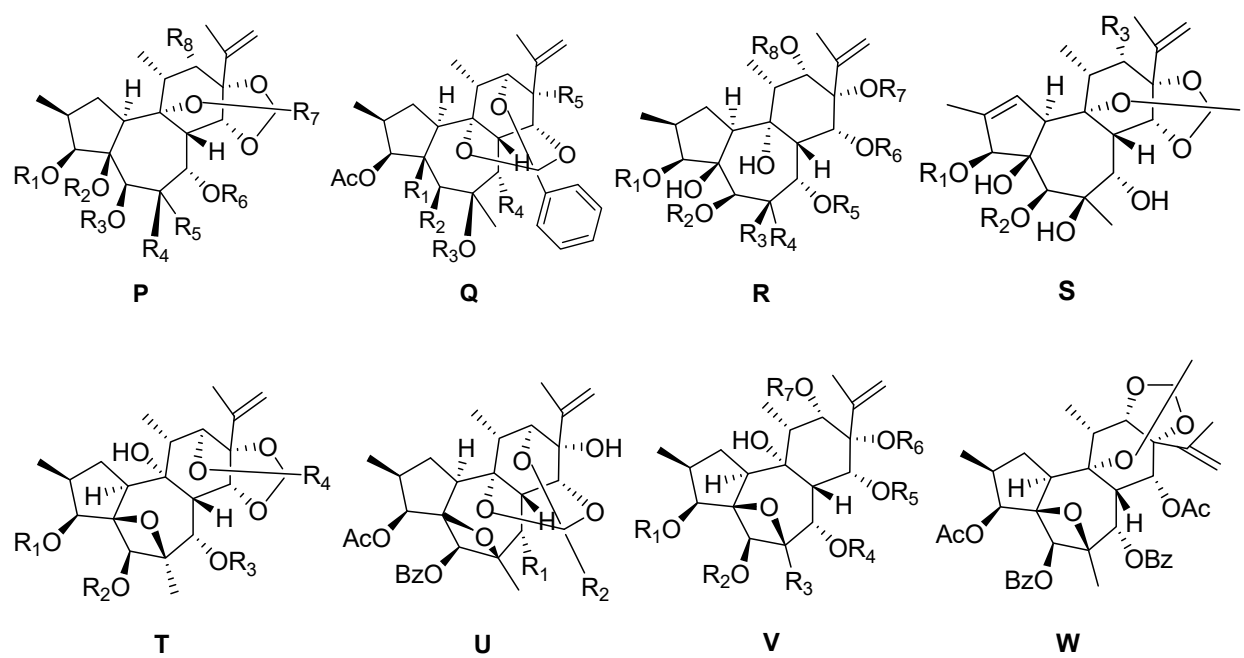

Figure 4. Eight types $(\mathbf{P}-\mathbf{W})$ of genkwanines skeletons. 
Table 4. Reported structures of genkwanines skeletons.

\begin{tabular}{|c|c|c|c|c|c|c|c|c|c|c|}
\hline No. & Name & $\mathbf{R}_{1}$ & $\mathbf{R}_{2}$ & $\mathbf{R}_{3}$ & $\mathbf{R}_{4}$ & $\mathbf{R}_{5}$ & $\mathbf{R}_{6}$ & $\mathbf{R}_{7}$ & $\mathbf{R}_{8}$ & Type \\
\hline 88 & Genkwanine A & $\begin{array}{c}\mathrm{H} \\
\mathrm{CO}(\mathrm{CH}=\end{array}$ & $\mathrm{H}$ & $\mathrm{H}$ & $\mathrm{OH}$ & $\mathrm{CH}_{2} \mathrm{OH}$ & $\mathrm{H}$ & $\mathrm{Ph}$ & $\mathrm{H}$ & $\mathrm{P}$ \\
\hline 89 & Genkwanine B & $\begin{array}{c}\mathrm{CH})_{2}\left(\mathrm{CH}_{2}\right)_{4} \\
\mathrm{CH}_{3}\end{array}$ & $\mathrm{H}$ & $\mathrm{H}$ & $\mathrm{OH}$ & $\mathrm{CH}_{2} \mathrm{OH}$ & $\mathrm{H}$ & $\mathrm{Ph}$ & $\mathrm{H}$ & $\mathrm{P}$ \\
\hline 90 & Genkwanine C & $\begin{array}{c}\mathrm{CO}(\mathrm{CH}= \\
\mathrm{CH})_{3}\left(\mathrm{CH}_{2}\right)_{2} \\
\mathrm{CH}_{3}\end{array}$ & $\mathrm{H}$ & $\mathrm{H}$ & $\mathrm{OH}$ & $\mathrm{CH}_{2} \mathrm{OH}$ & $\mathrm{H}$ & $\mathrm{Ph}$ & $\mathrm{H}$ & $\mathrm{P}$ \\
\hline 93 & Genkwanine F & $\mathrm{H}$ & $\mathrm{H}$ & $\mathrm{H}$ & $\mathrm{OH}$ & $\begin{array}{c}\mathrm{CH}_{2} \mathrm{OCO}(\mathrm{CH}= \\
\mathrm{CH})_{2}\left(\mathrm{CH}_{2}\right)_{4} \mathrm{CH}_{3} \\
\mathrm{CH}_{2} \mathrm{COO}(\mathrm{C}\end{array}$ & $\mathrm{H}$ & $\mathrm{Ph}$ & $\mathrm{H}$ & $\mathrm{P}$ \\
\hline 94 & Genkwanine G & $\mathrm{H}$ & $\mathrm{H}$ & $\mathrm{H}$ & $\mathrm{OH}$ & $\begin{array}{c}\mathrm{H}=\mathrm{CH}) \\
\left(\mathrm{CH}_{2}\right)_{6} \mathrm{CH}_{3}\end{array}$ & $\mathrm{H}$ & $\mathrm{Ph}$ & $\mathrm{H}$ & $\mathrm{P}$ \\
\hline 99 & Trigothysoid G & Ac & $\mathrm{H}$ & $\mathrm{Bz}$ & $\mathrm{OH}$ & ME & $\mathrm{H}$ & $\mathrm{Me}$ & $\mathrm{OBz}$ & $\mathrm{P}$ \\
\hline 100 & Trigoxyphin $\mathrm{H}$ & $\mathrm{Ac}$ & $\mathrm{H}$ & Ac & OCOPh & $\mathrm{Me}$ & Ac & $\mathrm{Ph}$ & OAc & $\mathrm{P}$ \\
\hline 101 & Trigohownin D & Ac & $\mathrm{Bz}$ & Ac & $\mathrm{OH}$ & $\mathrm{Me}$ & Ac & $\mathrm{Ph}$ & OAc & $\mathrm{P}$ \\
\hline 102 & Trigohownin E & Ac & $\mathrm{H}$ & $\mathrm{Bz}$ & $\mathrm{OH}$ & $\mathrm{Me}$ & Ac & $\mathrm{Me}$ & $\mathrm{OBz}$ & $\mathrm{P}$ \\
\hline 103 & Trigoxyphin F & Ac & $\mathrm{H}$ & Ac & $\mathrm{OBz}$ & $\mathrm{Me}$ & Ac & $\mathrm{Ph}$ & $\mathrm{OH}$ & $\mathrm{P}$ \\
\hline 104 & Trigoxyphin I & Ac & $\mathrm{H}$ & Ac & OCOPh & $\mathrm{Me}$ & $\mathrm{Ac}$ & $\mathrm{Ph}$ & Ac & $\mathrm{P}$ \\
\hline 105 & Trigoxyphin U & Ac & $\mathrm{H}$ & Ac & $\mathrm{Me}$ & OCOPh & $\mathrm{Ac}$ & $\mathrm{ME}$ & OCOPh & $\mathrm{P}$ \\
\hline 106 & Trigonosin C & $\mathrm{H}$ & $\mathrm{H}$ & $\mathrm{H}$ & $\mathrm{OH}$ & $\mathrm{Me}$ & $\mathrm{H}$ & $\mathrm{Ph}$ & $\mathrm{OBz}$ & $\mathrm{P}$ \\
\hline 107 & Trigonothyrin F & $\mathrm{H}$ & $\mathrm{H}$ & $\mathrm{H}$ & $\mathrm{OH}$ & $\mathrm{Me}$ & $\mathrm{H}$ & $\mathrm{Ph}$ & $\mathrm{H}$ & $\mathrm{P}$ \\
\hline 108 & Trigohownin A & OAc & $\mathrm{OH}$ & $\mathrm{Bz}$ & OAc & $\mathrm{OH}$ & - & - & - & Q \\
\hline 109 & Trigohownin B & $\mathrm{OBz}$ & $\mathrm{OAC}$ & $\mathrm{H}$ & OAc & $\mathrm{OH}$ & - & - & - & $\hat{Q}$ \\
\hline 110 & Trigohownin C & $\mathrm{OH}$ & OAC & $\mathrm{Bz}$ & $\mathrm{OH}$ & $\mathrm{OH}$ & - & - & - & $\hat{Q}$ \\
\hline 114 & Genkwanine J & $\mathrm{H}$ & $\mathrm{H}$ & $\mathrm{OH}$ & $\begin{array}{c}\mathrm{CH})_{2}(\mathrm{C} \\
\left.\mathrm{H}_{2}\right)_{4} \mathrm{CH}_{3}\end{array}$ & $\mathrm{H}$ & $\mathrm{Bz}$ & $\mathrm{H}$ & $\mathrm{H}$ & $\mathrm{R}$ \\
\hline 115 & Genkwanine K & $\mathrm{H}$ & $\mathrm{H}$ & $\mathrm{OH}$ & $\mathrm{CH}_{2} \mathrm{Bz}$ & $\mathrm{H}$ & $\mathrm{Bz}$ & $\mathrm{H}$ & $\mathrm{H}$ & $\mathrm{R}$ \\
\hline 116 & Trigoxyphin W & Ac & Ac & $\mathrm{Me}$ & OCOPh & $\mathrm{H}$ & $\mathrm{H}$ & $\mathrm{COPh}$ & $\mathrm{H}$ & $\mathrm{R}$ \\
\hline 117 & Trigohownin F & Ac & Ac & $\mathrm{OBz}$ & $\mathrm{Me}$ & $\mathrm{Ac}$ & $\mathrm{H}$ & $\mathrm{Bz}$ & $\mathrm{OH}$ & $\mathrm{R}$ \\
\hline 118 & Trigohownin G & Ac & Ac & $\mathrm{OBz}$ & $\mathrm{Me}$ & $\mathrm{Ac}$ & Ac & $\mathrm{Bz}$ & $\mathrm{OH}$ & $\mathrm{R}$ \\
\hline 119 & Trigohownin $\mathrm{H}$ & Ac & Ac & $\mathrm{OBz}$ & $\mathrm{Me}$ & Ac & $\mathrm{Bz}$ & Ac & $\mathrm{OH}$ & $\mathrm{R}$ \\
\hline 120 & Trigohownin I & Ac & $\mathrm{Bz}$ & $\mathrm{OH}$ & $\mathrm{Me}$ & Ac & Ac & $\mathrm{Bz}$ & $\mathrm{OH}$ & $\mathrm{R}$ \\
\hline 121 & Trigonothyrin G & Ac & $\mathrm{H}$ & $\mathrm{OCOPh}$ & - & - & - & - & - & $S$ \\
\hline 122 & Trigothysoid A & $\mathrm{H}$ & $\mathrm{H}$ & $\mathrm{OBz}$ & - & - & - & - & - & $\mathrm{S}$ \\
\hline 123 & Trigothysoid B & Ac & $\mathrm{Bz}$ & $\mathrm{OBz}$ & - & - & - & - & - & $\mathrm{S}$ \\
\hline 124 & Trigothysoid C & $\mathrm{H}$ & $\mathrm{Ac}$ & $\mathrm{OBz}$ & - & - & - & - & - & $\mathrm{S}$ \\
\hline 125 & Trigonothyrin A & $\mathrm{Bz}$ & Ac & $\mathrm{Bz}$ & $\mathrm{Me}$ & - & - & - & - & $\mathrm{T}$ \\
\hline 126 & Trigonothyrin B & $\mathrm{H}$ & $\mathrm{Bz}$ & $\mathrm{Bz}$ & $\mathrm{Me}$ & - & - & - & - & $\mathrm{T}$ \\
\hline 127 & Trigonothyrin C & Ac & $\mathrm{Bz}$ & $\mathrm{Bz}$ & $\mathrm{Me}$ & - & - & - & - & $\mathrm{T}$ \\
\hline 128 & Trigothysoid L & $\mathrm{Ac}$ & $\mathrm{Bz}$ & Ac & $\mathrm{Ph}$ & - & - & - & - & $\mathrm{T}$ \\
\hline 129 & Trigonostempene B & Ac & Ac & $\mathrm{Bz}$ & $\mathrm{Me}$ & - & - & - & - & $\mathrm{T}$ \\
\hline 130 & Trigochinin C & OAc & $\mathrm{Ph}$ & - & - & - & - & - & - & $\mathrm{U}$ \\
\hline 131 & Trigothysoid K & $\mathrm{OBz}$ & $\mathrm{Me}$ & - & - & - & - & - & - & $\mathrm{U}$ \\
\hline 132 & Trigolins A & $\mathrm{H}$ & $\mathrm{Bz}$ & $\mathrm{Me}$ & Ac & Ac & $\mathrm{H}$ & $\mathrm{Bz}$ & - & $\mathrm{V}$ \\
\hline 133 & Trigolins B & Ac & $\mathrm{Bz}$ & $\mathrm{Me}$ & Ac & $\mathrm{H}$ & $\mathrm{H}$ & $\mathrm{Bz}$ & - & $\mathrm{V}$ \\
\hline 134 & Trigolins C & Ac & $\mathrm{Bz}$ & $\mathrm{Me}$ & Ac & $\mathrm{Bz}$ & $\mathrm{H}$ & $\mathrm{H}$ & - & $\mathrm{V}$ \\
\hline 135 & Trigolins D & Ac & $\mathrm{Bz}$ & $\mathrm{Me}$ & Ac & Ac & $\mathrm{H}$ & $\mathrm{Bz}$ & - & $\mathrm{V}$ \\
\hline 136 & Trigolins E & Ac & $\mathrm{Bz}$ & $\mathrm{Me}$ & $\mathrm{Bz}$ & Ac & $\mathrm{H}$ & Ac & - & $\mathrm{V}$ \\
\hline 137 & Trigolins F & Ac & Ac & $\mathrm{Me}$ & $\mathrm{Bz}$ & $\mathrm{Ac}$ & $\mathrm{H}$ & $\mathrm{Bz}$ & - & $\mathrm{V}$ \\
\hline 138 & Trigolins G & $\mathrm{H}$ & $\mathrm{Bz}$ & $\mathrm{Me}$ & $\mathrm{Bz}$ & $\mathrm{AC}$ & $\mathrm{H}$ & $\mathrm{Bz}$ & - & $\mathrm{V}$ \\
\hline 139 & Trigothysoid H & Ac & Ac & $\mathrm{CH}_{2} \mathrm{OAc}$ & $\mathrm{Ac}$ & Ac & $\mathrm{Bz}$ & $\mathrm{Ac}$ & - & $\mathrm{V}$ \\
\hline 140 & Trigothysoid I & Ac & Ac & $\mathrm{CH}_{2} \mathrm{OAc}$ & Ac & $\mathrm{Ac}$ & $\mathrm{H}$ & $\mathrm{Bz}$ & - & $\mathrm{V}$ \\
\hline 141 & Trigothysoid J & Ac & $\mathrm{Bz}$ & $\mathrm{Me}$ & $\mathrm{Ac}$ & $\mathrm{Ac}$ & $\mathrm{H}$ & $\mathrm{Bz}$ & - & V \\
\hline 142 & Trigonosin D & $\mathrm{H}$ & $\mathrm{H}$ & $\mathrm{Me}$ & Ac & $\mathrm{Ac}$ & $\mathrm{COPh}$ & Ac & - & $\mathrm{V}$ \\
\hline 143 & Trigonothyrin D & Ac & Ac & $\mathrm{Me}$ & Ac & Ac & $\mathrm{COPh}$ & Ac & - & $\mathrm{V}$ \\
\hline 144 & Trigonothyrin E & $\mathrm{H}$ & Ac & $\mathrm{Me}$ & Ac & $\mathrm{Ac}$ & $\mathrm{COPh}$ & Ac & - & $\mathrm{V}$ \\
\hline 145 & Trigochinin A & $\mathrm{H}$ & $\mathrm{Bz}$ & $\mathrm{Me}$ & Ac & $\mathrm{Ac}$ & $\mathrm{COPh}$ & $\mathrm{Ac}$ & - & V \\
\hline 146 & Trigochinin B & Ac & $\mathrm{Bz}$ & $\mathrm{Me}$ & Ac & $\mathrm{Ac}$ & $\mathrm{COPh}$ & Ac & - & $\mathrm{V}$ \\
\hline 147 & Trigochinin D & $\mathrm{H}$ & $\mathrm{Bz}$ & $\mathrm{Me}$ & Ac & Ac & $\mathrm{Bz}$ & Ac & - & $\mathrm{V}$ \\
\hline 148 & Trigochinin E & Ac & $\mathrm{Bz}$ & $\mathrm{Me}$ & $\mathrm{Ac}$ & $\mathrm{Ac}$ & $\mathrm{Bz}$ & Ac & - & $\mathrm{V}$ \\
\hline 149 & Trigochinin F & Ac & Ac & Ac & Ac & $\mathrm{Ac}$ & $\mathrm{Bz}$ & Ac & - & $\mathrm{V}$ \\
\hline 150 & Trigonostempene A & - & - & - & - & - & - & - & - & W \\
\hline
\end{tabular}




\subsection{1-Alkyldaphnanes}

1-alkyldaphnanes have a large ring between the end of the orthoester alkyl chain and C-1 of ring A (Figure 5, Table 5). Pimelea factors $S_{6}(\mathbf{1 6 8})$ and $S_{7}(\mathbf{1 6 9})$ have been isolated from the flower buds of Wikstroemiachamaedaphne and have shown moderate cytotoxic activities against human myeloid leukemia HL-60, hepatocellular carcinoma SMMC-7721, lung cancer A549, breast cancer MCF-7, and colon cancer SW480 [1]. Compound pimelea factor $\mathrm{P}_{2}$ (155) has been obtained from the fresh bark of Wikstroemiaretusa, and has been shown to exhibit cytotoxicity in 10 cell lines (including HeLa, HepG2, HT-1080, HCT116, A375-S2, MCF-7, A549, U-937, K562 and HL60 cell lines) [14]. Wikstroelides E-G, K and $\mathrm{O}$ (163-167) have been isolated from the fresh bark of Wikstroemiaretusa. Among them, compound wikstroelide $\mathrm{E}$ has been shown to exhibit the highest activity against cell lines PC-6 (human lung cancer cell line) and P388 (mouse leukaemia cell line), followed by wikstroelides A and J, which have the orthoester group without a fatty acid at the 20-hydroxyl [12]. Compounds stelleralides A-C (151-152, 174) and gnidimacrin (153) have been isolated from the Stellerachamaejasme L. [5]. Genkwadane B (154), pimelotides $A$ and $C(\mathbf{1 7 0}, \mathbf{1 7 2})$, and genkwadane $C(\mathbf{1 5 6})$ have been isolated from the flower buds of Daphne genkwa [14]. Compounds wikstroelides R-T (157-159) have been obtained from the flower buds of Wikstroemiachamaedaphne. Wikstroelide $\mathrm{R}$ has been shown to have moderate cytotoxic activities against human cancer cell lines [1]. Compounds kirkinines B, C, and E (160-162) were isolated from Synaptolepiskirkii. Pimelotides B and D $(\mathbf{1 7 1}, \mathbf{1 7 3})$ have beenobtained from Pelongata [40].

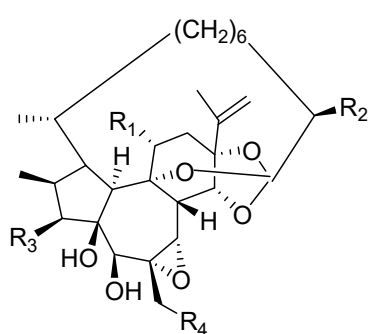

X1

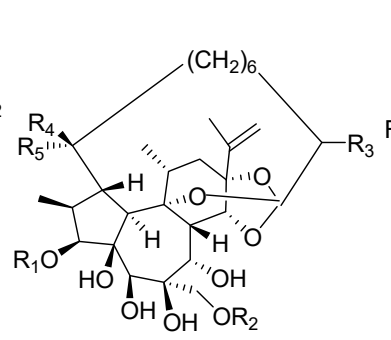

$\mathrm{X} 2$

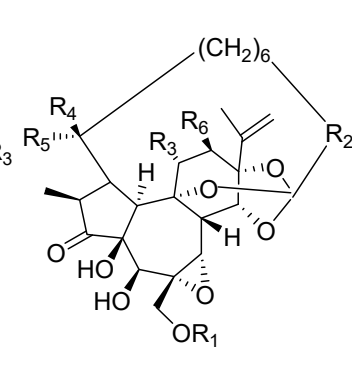

$\mathbf{X} 3$

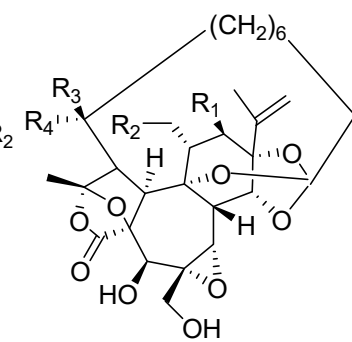

X4

Figure 5. Four types (X1-X4) of 1-alkyldaphnanes skeletons.

Table 5. Reported structures of 1-alkyldaphnanes skeletons.

\begin{tabular}{|c|c|c|c|c|c|c|c|c|}
\hline No. & Name & $\mathbf{R}_{1}$ & $\mathbf{R}_{2}$ & $\mathbf{R}_{3}$ & $\mathbf{R}_{4}$ & $\mathbf{R}_{5}$ & $\mathbf{R}_{6}$ & Type \\
\hline 151 & Stelleralide A & $\mathrm{CH}_{2} \mathrm{OAc}$ & $\mathrm{OH}$ & $\mathrm{OBz}$ & $\mathrm{OH}$ & - & - & $\mathrm{X} 1$ \\
\hline 152 & Stelleralide B & $\mathrm{CH}_{2} \mathrm{OBz}$ & $\mathrm{H}$ & $\mathrm{OBz}$ & $\mathrm{OH}$ & - & - & $\mathrm{X} 1$ \\
\hline 153 & Gnidimacrin & $\mathrm{CH}_{2} \mathrm{OBz}$ & $\mathrm{OH}$ & $\mathrm{OBz}$ & $\mathrm{OH}$ & - & - & $\mathrm{X} 1$ \\
\hline 154 & Genkwadane B & $\mathrm{Me}$ & $\mathrm{H}$ & $\mathrm{OH}$ & $\mathrm{OBz}$ & - & - & $\mathrm{X} 1$ \\
\hline 155 & Pimelea factor $\mathrm{P}_{2}$ & $\mathrm{CH}_{2} \mathrm{OH}$ & $\mathrm{H}$ & $\mathrm{OBz}$ & $\mathrm{OH}$ & - & - & $\mathrm{X} 1$ \\
\hline 156 & Genkwadane C & $\mathrm{H}$ & benzoyl & $\mathrm{H}$ & $\mathrm{H}$ & $\mathrm{Me}$ & - & $\mathrm{X} 2$ \\
\hline 157 & Wikstroelide R & $\mathrm{H}$ & benzoyl & $\mathrm{OH}$ & $\mathrm{H}$ & $\mathrm{Me}$ & - & $\mathrm{X} 2$ \\
\hline 158 & Wikstroelide S & benzoyl & $\mathrm{H}$ & $\mathrm{H}$ & $\mathrm{Me}$ & $\mathrm{H}$ & - & $\mathrm{X} 2$ \\
\hline 159 & Wikstroelide T & $\mathrm{H}$ & trans-cinnamoyl & $\mathrm{H}$ & $\mathrm{H}$ & $\mathrm{Me}$ & - & $\mathrm{X} 2$ \\
\hline 160 & Kirkinine B & $\mathrm{H}$ & $\mathrm{CH}=\mathrm{CH}\left(\mathrm{CH}_{2}\right)_{5}$ & $\mathrm{Me}$ & $\mathrm{H}$ & $\mathrm{Me}$ & $\mathrm{H}$ & $\mathrm{X} 3$ \\
\hline 161 & Kirkinine C & $\mathrm{H}$ & $\mathrm{CH}=\mathrm{CH}\left(\mathrm{CH}_{2}\right)_{5}$ & $\mathrm{Me}$ & $\mathrm{H}$ & $\mathrm{Me}$ & OAc & $\mathrm{X} 3$ \\
\hline 162 & Kirkinine E & $\mathrm{H}$ & $\mathrm{CH}=\mathrm{CH}\left(\mathrm{CH}_{2}\right)_{5}$ & $\mathrm{Me}$ & $\mathrm{OH}$ & $\mathrm{Me}$ & $\mathrm{H}$ & $\mathrm{X} 3$ \\
\hline 163 & Wikstroelide E & $\mathrm{H}$ & $\mathrm{CH}_{2}$ & $\mathrm{Me}$ & $\mathrm{H}$ & $\mathrm{Me}$ & $\mathrm{H}$ & $\mathrm{X} 3$ \\
\hline 164 & Wikstroelide F & $\mathrm{H}$ & $\mathrm{CH}_{2}$ & $\mathrm{CH}_{2} \mathrm{OBz}$ & $\mathrm{H}$ & $\mathrm{Me}$ & $\mathrm{H}$ & $\mathrm{X} 3$ \\
\hline 165 & Wikstroelide G & palmitic acid & $\mathrm{CH}_{2}$ & $\mathrm{CH}_{2} \mathrm{OBz}$ & $\mathrm{H}$ & $\mathrm{Me}$ & $\mathrm{H}$ & $\mathrm{X} 3$ \\
\hline 166 & Wikstroelide K & $\mathrm{CO}\left(\mathrm{CH}_{2}\right) 14 \mathrm{CH}_{3}$ & $\mathrm{CH}_{2}$ & $\mathrm{CH}_{2} \mathrm{OBz}$ & $\mathrm{Me}$ & $\mathrm{H}$ & $\mathrm{H}$ & $\mathrm{X} 3$ \\
\hline 167 & Wikstroelide $\mathrm{O}$ & $\mathrm{H}$ & $\mathrm{CH}_{2}$ & $\mathrm{CH}_{2} \mathrm{OBz}$ & $\mathrm{Me}$ & $\mathrm{H}$ & $\mathrm{H}$ & $\mathrm{X} 3$ \\
\hline 168 & Pimelea factor $S_{6}$ & $\mathrm{OH}$ & $\mathrm{CH}_{2}$ & $\mathrm{Me}$ & $\mathrm{H}$ & $\mathrm{Me}$ & $\mathrm{H}$ & $\mathrm{X} 3$ \\
\hline 169 & Pimelea factor $S_{7}$ & $\mathrm{OH}$ & $\mathrm{CH}_{2}$ & $\mathrm{Me}$ & $\mathrm{Me}$ & $\mathrm{H}$ & $\mathrm{H}$ & $\mathrm{X} 3$ \\
\hline 170 & Pimelotide A & $\mathrm{H}$ & $\mathrm{H}^{2}$ & $\mathrm{Me}$ & $\mathrm{H}$ & - & - & $\mathrm{X} 4$ \\
\hline 171 & Pimelotide B & OAc & $\mathrm{H}$ & $\mathrm{H}$ & $\mathrm{Me}$ & - & - & $\mathrm{X} 4$ \\
\hline 172 & Pimelotide C & $\mathrm{H}$ & $\mathrm{H}$ & $\mathrm{H}$ & $\mathrm{Me}$ & - & - & $\mathrm{X} 4$ \\
\hline 173 & Pimelotide D & OAc & $\mathrm{H}$ & $\mathrm{Me}$ & $\mathrm{H}$ & - & - & $\mathrm{X} 4$ \\
\hline 174 & Stelleralide C & $\mathrm{H}$ & $\mathrm{OBz}$ & $\mathrm{Me}$ & $\mathrm{H}$ & - & - & $\mathrm{X} 4$ \\
\hline
\end{tabular}




\subsection{Rediocides}

Rediocides usually have a 12-carbon macrolide structure between C-3 and C-16, and have a special C-9, C-12, and C-14 orthoester structure (Figure 6, Table 6). The active compounds trigothysoids N-P (182-184), rediocides A, C, and F (176-177, 179), and trigonosin F (181) have been obtained from the twigs and leaves of Trigonostemonthyrsoideum. Amongst them, compounds trigothysoid N, rediocides A, $\mathrm{C}$, and $\mathrm{F}$, and trigonosins $\mathrm{F}$ have shownpotent anti-HIV-1 activity, with $\mathrm{EC}_{50}$ values ranging from 0.001 to $0.015 \mathrm{nM}$. Additionally, trigothysoid $\mathrm{O}$ has been shown to exhibit moderate anti-HIV-1 activity [11], while rediocide $\mathrm{A}$ has shown potent activities against mosquito larvae in an in-vitro assay study and against fleas (Ctenocephalides felis) in an artificial membrane feeding system, exhibiting $\mathrm{LD}_{90}$ values of 1 and 0.25 ppm, respectively [39]. Trigochilides A and B $(\mathbf{1 7 5}, \mathbf{1 8 6})$ have been isolated from the twigs and leaves of Trigonostemonchinensis Merr. Trigochilide A has shown modest cytotoxicity against HL-60 (human leukemia) and BEL-7402 (human hepatoma), with demonstrated $\mathrm{IC}_{50}$ values of 3.68 and $8.22 \mu \mathrm{M}$, respectively, whereas compound trigochilide $\mathrm{B}$ has only been shown to exhibit weak cytotoxicity against two tumor cell lines, with $\mathrm{IC}_{50}$ values of 33.35 and $54.85 \mu \mathrm{M}$ [1]. Compound rediocide $\mathrm{E}$ (178) has been obtained from the roots of Trigonostemonreidioides, and has shown significant acaricidal activity on D. pteronyssinus [40]. Trigonosin E (180) and trigonostempene D (185) have beenisolated from the twigs and leaves of Trigonostemonthyrsoideum [16,21]. Rediocides B, G, and D (187-189) have been isolated from the Trigonostemonreidioides, and have been evaluated for their insecticidal properties in an anti-flea artificial membrane feeding assay (as detailed earlier). In this assay, rediocides B and D exhibited $L_{90}$ values of 0.25 and 0.5 ppm, respectively, and thus were equipotent with rediocide $\mathrm{A}$ $\left(\mathrm{LD}_{90} 0.25 \mathrm{ppm}\right)$ [41].

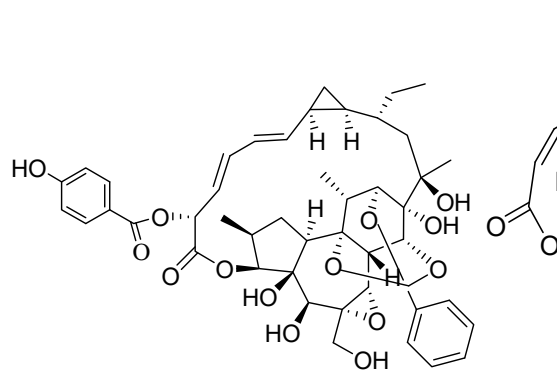

Y1

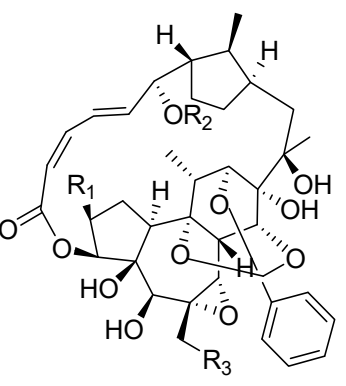

Y2

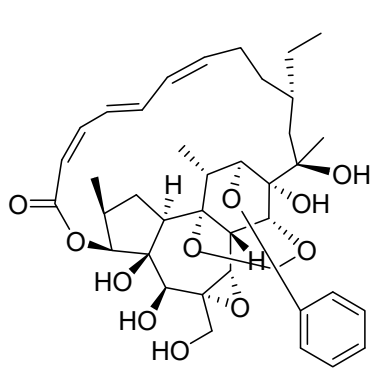

Y3

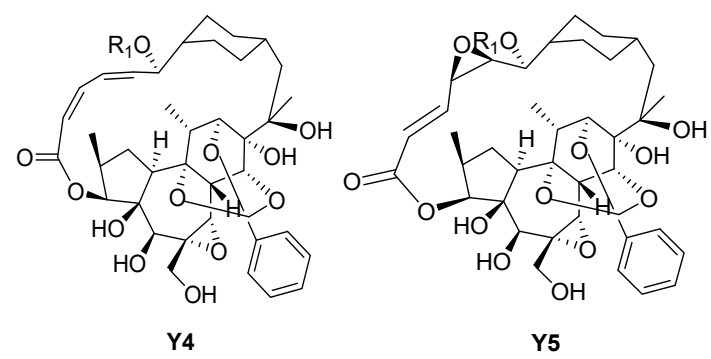

Figure 6. Five types (Y1-Y5) of rediocides skeletons.

Table 6. Reported structures of rediocides skeletons.

\begin{tabular}{cccccc}
\hline No. & Name & $\mathbf{R}_{\mathbf{1}}$ & $\mathbf{R}_{\mathbf{2}}$ & $\mathbf{R}_{\mathbf{3}}$ & Type \\
\hline 175 & Trigochilide A & - & - & - & $\mathrm{Y} 1$ \\
176 & Rediocide A & $\mathrm{Me}$ & $\mathrm{COCH}_{2} \mathrm{CH}\left(\mathrm{CH}_{3}\right)_{2}$ & $\mathrm{OH}$ & $\mathrm{Y} 2$ \\
177 & Rediocide C & $\mathrm{Me}$ & $\mathrm{Bz}$ & $\mathrm{OH}$ & $\mathrm{Y} 2$ \\
178 & Rediocide E & $\mathrm{H}$ & $\mathrm{COCH}_{2} \mathrm{CH}\left(\mathrm{CH}_{3}\right)_{2}$ & $\mathrm{OH}$ & $\mathrm{Y} 2$ \\
179 & Rediocide F & $\mathrm{H}$ & $\mathrm{Bz}$ & $\mathrm{OH}$ & $\mathrm{Y} 2$ \\
180 & Trigonosin E & $\mathrm{Me}$ & $\mathrm{COPh}$ & $\mathrm{OH}$ & $\mathrm{Y} 2$ \\
181 & Trigonosin F & $\mathrm{Me}$ & $\mathrm{COPh}$ & $\mathrm{OH}$ & $\mathrm{Y} 2$ \\
\hline
\end{tabular}


Table 6. Cont.

\begin{tabular}{cccccc}
\hline No. & Name & $\mathbf{R}_{\mathbf{1}}$ & $\mathbf{R}_{\mathbf{2}}$ & $\mathbf{R}_{\mathbf{3}}$ & Type \\
\hline 182 & Trigothysoid N & $\mathrm{Me}$ & $\mathrm{COCH}{ }_{2} \mathrm{CH}\left(\mathrm{CH}_{3}\right)_{2}$ & $\mathrm{OH}$ & $\mathrm{Y} 2$ \\
183 & Trigothysoid O & $\mathrm{Me}$ & $\mathrm{COPh}$ & $\mathrm{H}$ & $\mathrm{Y} 2$ \\
184 & Trigothysoid P & $\mathrm{Me}$ & $\mathrm{COCH}_{2} \mathrm{CH}\left(\mathrm{CH}_{3}\right)_{2}$ & $\mathrm{H}$ & $\mathrm{Y} 2$ \\
185 & Trigonostempene D & $\mathrm{Me}$ & $\mathrm{Val}$ & $\mathrm{H}$ & $\mathrm{Y} 2$ \\
186 & Trigochilide B & - & - & - & $\mathrm{Y} 3$ \\
187 & Rediocide B & $\mathrm{COCH}_{2} \mathrm{CH}\left(\mathrm{CH}_{3}\right)_{2}$ & - & - & $\mathrm{Y} 4$ \\
188 & Rediocide G & $\mathrm{Bz}$ & - & - & $\mathrm{Y} 4$ \\
189 & Rediocide D & $\mathrm{COCH}_{2} \mathrm{CH}\left(\mathrm{CH}_{3}\right)_{2}$ & - & - & $\mathrm{Y} 5$ \\
\hline
\end{tabular}

\section{Conclusions}

It can be concluded that the bioactive activities of daphnane-type diterpenoids is obviously related to structure types. The most important points of them are the following: (1) The orthoester groups at C-9, C-13 and C-14 are essential to the cytotoxic activity. Daphnane-type diterpenoids with orthoester groups at C-9, C-13, and C-14 usually have stronger activity than daphnane-type diterpenoids with orthoester groups at C-9, C-12, and C-14 or C-12, C-13 and C-14. The absence of the orthoester group is unhelpful to the cytotoxic activity. (2) Specific to the 6-epoxyl groups, free 20-hydroxyl and 3-carbonyl are important for their activities. (3) Side chains at C-10 are crucial for cytotoxic activities. Generally speaking, long C-10 alkyl chains are more important than phenyl at C-10. Interestingly, the structure with macro-lactones exhibited much stronger activity than the others. Due to the rich activities of daphnane-type diterpenoids, researchers have not stopped exploring and researching such compounds and their bioactive activities from plants.

Funding: This research was funded by the National Natural Science Foundation of China [grant number 81860759].

Conflicts of Interest: There is no conflict of interest associated with the authors of this paper.

\section{References}

1. Jayasuriya, H.; Zink, D.L.; Singh, S.B.; Borris, R.P.; Nanakorn, W.; Beck, H.T.; Balick, M.J.; Goetz, M.A.; Slayton, L.; Gregory, L.; et al. Structure and Stereochemistry of Rediocide A, a Highly Modified Daphnane from Trigonostemon reidioides Exhibiting Potent Insecticidal Activity. ChemInform 2000, 31, 4998-4999.

2. Carney, J.R.; Krenisky, J.M.; Williamson, R.T.; Luo, J.; Carlson, T.J.; Hsu, V.L.; Moswa, J.L. Maprouneacin, a new daphnane diterpenoid with potent antihyperglycemic activity from Maprounea africana. J. Nat. Prod. 1999, 62, 345-347. [CrossRef] [PubMed]

3. He, W.; Cik, M.; Lesage, A. Kirkinine, a new daphnane orthoester with potent neurotrophic activity from Synaptolepis kirkii. J. Nat. Prod. 2000, 63, 1185-1187. [CrossRef] [PubMed]

4. He, W.; Cik, M.; Appendino, G. Daphnane-type diterpene orthoesters and their biological activities. Mini-Rev. Med. Chem. 2002, 2, 185-200. [CrossRef] [PubMed]

5. Asada, Y.; Sukemori, A.; Watanabe, T.; Malla, K.J.; Yoshikawa, T.; Li, W.; Koike, K.; Chen, C.H.; Akiyama, T.; Qian, K.; et al. Stelleralides A-C, Novel Potent Anti-HIV Daphnane-Type Diterpenoids from Stellera chamaejasme L. Org. Lett. 2011, 13, 2904-2907. [CrossRef] [PubMed]

6. Zhang, L.; Luo, R.H.; Wang, F.; Dong, Z.J.; Yang, L.M.; Zheng, Y.T.; Liu, J.K. Daphnane diterpenoids isolated from Trigonostemon thyrsoideum as HIV-1 antivirals. Phytochemistry 2010, 71, 1879-1883. [CrossRef] [PubMed]

7. Chen, S.K.; Chen, B.Y.; Li, H. Flora of China (Zhongguo Zhiwu Zhi); Science: Beijing, China, 1997; Volume 44, pp. 162-170.

8. Zhang, L.; Luo, R.H.; Wang, F.; Jiang, M.Y.; Dong, Z.J.; Yang, L.M.; Zheng, Y.T.; Liu, J.K. Highly functionalized daphnane diterpenoids from Trigonostemon thyrsoideum. Org. Lett. 2010, 12, 152-155. [CrossRef]

9. Wang, H.B.; Liu, L.P.; Wang, X.Y. ${ }^{13}$ C-NMR data of daphnane diterpenoids. Magn. Reson. Chem. 2013, 51, 580-592. [CrossRef] 
10. Huang, S.Z.; Zhang, X.J.; Li, X.Y.; Kong, L.M.; Jiang, H.J.; Ma, Q.Y.; Liu, Y.Q.; Hu, J.M.; Zheng, Y.T.; Li, Y. Daphnane-type diterpene esters with cytotoxic and anti-HIV-1 activities from Daphne acutiloba Rehd. Phytochemistry 2012, 75, 99-107. [CrossRef]

11. Cheng, Y.Y.; Chen, H.; He, H.P.; Zhang, Y.; Li, S.F.; Tang, G.H.; Guo, L.L.; Yang, W.; Zhu, F.; Zheng, Y.T. Anti-HIV active daphnane diterpenoids from Trigonostemon thyrsoideum. Phytochemistry 2013, 96, 360-369. [CrossRef]

12. Abe, F.; Iwase, Y.; Yamauchi, T.; Kinjo, K.; Yaga, S.; Ishii, M.; Iwahana, M. Minor daphnane-type diterpenoids from Wikstroemia retusa. Phytochemistry 1998, 47, 833-837. [CrossRef]

13. Li, L.Z.; Gao, P.Y.; Peng, Y.; Wang, L.H.; Song, S.J. A novel daphnane-type diterpene from the flower bud of Daphne genkwa. Chem. Nat. Compd. 2010, 46, 380-382. [CrossRef]

14. Li, F.; Sun, Q.; Hong, L.; Li, L.; Wu, Y.; Xia, M.; Ikejima, T.; Peng, Y.; Song, S. Daphnane-type diterpenes with inhibitory activities against human cancer cell lines from Daphne genkwa. Bioorg. Med. Chem. Lett. 2013, 23, 2500-2504. [CrossRef] [PubMed]

15. Liu, F.; Yang, X.; Ma, J.; Yang, Y.; Xie, C.; Tuerhong, M.; Jin, D.Q.; Xu, J.; Lee, D.; Ohizumi, Y.; et al. Nitric oxide inhibitory daphnane diterpenoids as potential anti-neuroinflammatory agents for AD from the twigs of Trigonostemon thyrsoideus. Bioorg. Chem. 2017, 75, 149-156. [CrossRef] [PubMed]

16. Jo, S.K.; Hong, J.Y.; Park, H.J.; Lee, S.K. Anticancer Activity of Novel Daphnane Diterpenoids from Daphne genkwa through Cell-Cycle Arrest and Suppression of Akt/STAT/Src Signalings in Human Lung Cancer Cells. Biomol. Ther. (Seoul). 2012, 20, 513-519. [CrossRef]

17. Taninaka, H.; Takaishi, Y.; Honda, G.; Imakura, Y.; Sezik, E.; Yesilada, E. Terpenoids and aromatic compounds from Daphne oleoides ssp. oleoides. Phytochemistry (Oxford) 1999, 52, 1525-1529. [CrossRef]

18. Yu, L.; Zuo, W.J.; Mei, W.L.; Guo, Z.K.; Li, X.N.; Dai, H.F. Three new terpenoids from Trigonostemon xyphophylloides (Croiz.) L.K. Dai and T.L. Wu. Phytochem. Lett. 2013, 6, 472-475. [CrossRef]

19. Chen, Y.Y.; Guo, J.M.; Qian, Y.F.; Guo, S.; Ma, C.H.; Duan, J.A. Toxicity of daphnane-type diterpenoids from Genkwa Flos and their pharmacokinetic profile in rat. Phytomedicine 2013, 21, 82-89. [CrossRef]

20. Li, S.F.; Di, Y.T.; Li, S.L.; Zhang, Y.; Yang, F.M.; Sun, Q.Y.; Simo, J.M.; He, H.P.; Hao, X.J. Trigonosins A-F, Daphnane Diterpenoids from Trigonostemon thyrsoideum. J. Nat. Prod. 2011, 74, 464-469. [CrossRef]

21. Miyamae, Y.; Villareal, M.O.; Abdrabbah, M.B.; Isoda, H.; Shigemori, H. Hirseins A and B, Daphnane Diterpenoids from Thymelaea hirsuta That Inhibit Melanogenesis in B16 Melanoma Cells. J. Nat. Prod. 2009, 72, 938-941. [CrossRef]

22. Tchinda, A.Y.; Tsopmo, A.; Tene, M.; Kamnaing, P.; Ngnokam, D.; Tane, P.; Ayafor, J.F.; Connolly, J.D.; Farrugia, L.J. Diterpenoids from Neoboutonia glabrescens (Euphorbiaceae). Phytochemistry 2003, 64, 549-553. [CrossRef]

23. Hayes, P.Y.; Chow, S.; Somerville, M.J.; Fletcher, M.T. Daphnane- and Tigliane-Type Diterpenoid Esters and Orthoesters from Pimelea elongata. J. Nat. Prod. 2010, 73, 1907-1913. [CrossRef] [PubMed]

24. Lin, B.D.; Han, M.L.; Ji, Y.C.; Chen, H.D.; Yang, S.P.; Zhang, S.; Geng, M.Y.; Yue, J.M. Trigoxyphins A-G: diterpenes from Trigonostemon xyphophylloides. J. Nat. Prod. 2010, 73, 1301-1305. [CrossRef] [PubMed]

25. Powell, R.G.; Weisleder, D.; Smith, C.R. Daphnane Diterpenes from Diarthron vesiculosum: Vesiculosin and Isovesiculosin. J. Nat. Prod. 1985, 48, 102-107. [CrossRef]

26. Su, J.; Wu, Z. A New Daphnane-Type Diterpenoid from Daphne giraldii. Chem. Nat. Compd. 2014, 50, $285-287$. [CrossRef]

27. Chen, H.D.; Yang, S.P.; He, X.F.; Liu, H.B.; Ding, J.; Yue, J.M. Trigochinins D-I: six new daphnane-type diterpenoids from Trigonostemon chinensis. Tetrahedron 2010, 66, 5065-5070. [CrossRef]

28. Wei, Y.L.; Yu, Z.L.; Huo, X.K.; Tian, X.G.; Feng, L.; Huang, S.S.; Deng, S.; Ma, X.C.; Jia, J.M.; Wang, C. Diterpenoids from the roots of Euphorbia fischeriana and their inhibitory effects on alpha-glucosidase. J. Asian Nat. Prod. Res. 2018, 20, 977-984. [CrossRef]

29. Bang, K.K.; Yun, C.Y.; Lee, C.; Jin, Q.; Lee, J.W.; Jung, S.H.; Lee, D.; Lee, M.K.; Hong, J.T.; Kim, Y.; et al. Melanogenesis inhibitory daphnane diterpenoids from the flower buds of Daphne genkwa. Bioorg. Med. Chem. Lett. 2013, $23,3334$. [CrossRef]

30. Zhan, Z.J.; Fan, C.Q.; Ding, J.; Yue, J.M. Novel diterpenoids with potent inhibitory activity against endothelium cell HMEC and cytotoxic activities from a well-known TCM plant Daphne genkwa. Bioorg. Med. Chem. 2005, 13, 645-655. [CrossRef] 
31. Zhang, X.D.; Ni, W.; Yan, H.; Li, G.T.; Zhong, H.M.; Li, Y.; Liu, H.Y. Daphnane-Type Diterpenoid Glucosides and Further Constituents of Euphorbia pilosa. Chem. Biodivers. 2014, 11, 760-766. [CrossRef]

32. Yang, B.; Meng, Z.; Li, Z.; Sun, L.; Hu, Y.; Wang, Z.; Ding, G.; Xiao, W.; Han, C. Three daphnane diterpenoids from Trigonostemon xyphophylloides. Phytochem. Lett. 2015, 11, 270-274. [CrossRef]

33. Li, S.F.; Zhang, Y.; Huang, N.; Zheng, Y.T.; Di, Y.T.; Li, S.L.; Cheng, Y.Y.; He, H.P.; Hao, X.J. Daphnane diterpenoids from the stems of Trigonostemon lii and their anti-HIV-1 activity. Phytochemistry 2013, 93, $216-221$. [CrossRef]

34. Pejin, B.; Iodice, C.; Tommonaro, G. Synthesis and biological activities of thio-avarol derivatives. J. Nat. Prod. 2008, 71, 1850-1853. [CrossRef]

35. Tommonaro, G.; Vitale, R.M.; Pejin, B.; Iodice, C.; Canadas, S. Avarol derivatives as competitive AChE inhibitors, non hepatotoxic and neuroprotective agents for Alzheimer's disease. Eur. J. Med. Chem. 2016, 122, 326-338. [CrossRef]

36. Yang, B.; Chen, G.Y.; Song, X.P.; Yang, L.Q.; Han, C.R.; Wu, X.Y.; Li, X.M.; Zou, B.Y. Trigoxyphins H and I: Two new daphnane diterpenoids from Trigonostemon xyphophylloides. ChemInform 2012, 22, 3828-3830. [CrossRef]

37. Dong, S.H.; Zhang, C.R.; Xu, C.H.; Ding, J.; Yue, J.M. Daphnane-Type Diterpenoids from Trigonostemon howii. J. Nat. Prod. 2011, 74, 1255-1261. [CrossRef]

38. Guo, J.; Tian, J.; Yao, G.; Zhu, H.; Xue, Y.; Luo, Z.; Zhang, J.; Zhang, Y. Three new 1 $\alpha$-alkyldaphnane-type diterpenoids from the flower buds of Wikstroemia chamaedaphne. Fitoterapia 2015, 106, 242-246. [CrossRef]

39. Hayes, P.Y.; Chow, S.; Somerville, M.J.; Fletcher, M.T. Pimelotides A and B, Diterpenoid Ketal-Lactone Orthoesters with an Unprecedented Skeleton from Pimelea elongata. J. Nat. Prod. 2009, 72, 2081-2083. [CrossRef]

40. Chen, H.D.; He, X.F.; Ai, J.; Geng, M.Y.; Yue, J.M. Trigochilides A and B, Two Highly Modified Daphnane-Type Diterpenoids from Trigonostemon chinensis. Org. Lett. 2009, 11, 4080-4083. [CrossRef]

41. Jayasuriya, H.; Zink, D.L.; Borris, R.P.; Nanakorn, W.; Beck, H.T.; Balick, M.J.; Goetz, M.A.; Gregory, L.; Shoop, W.L.; Singh, S.B. Rediocides B-E, Potent Insecticides from Trigonostemon reidioides. J. Nat. Prod. 2004, 67, 228-231. [CrossRef]

Sample Availability: Samples of the compounds are available from the authors.

(C) 2019 by the authors. Licensee MDPI, Basel, Switzerland. This article is an open access article distributed under the terms and conditions of the Creative Commons Attribution (CC BY) license (http://creativecommons.org/licenses/by/4.0/). 